\title{
On Recognizing Virtual Honeypots and Countermeasures
}

\author{
Xinwen Fu, Wei Yu, Dan Cheng, Xuejun Tan, Kevin Streff, and Steve Graham
}

\begin{abstract}
Honeypots are decoys designed to trap, delay, and gather information about attackers. We can use honeypot logs to analyze attackers' behaviors and design new defenses. A virtual honeypot can emulate multiple honeypots on one physical machine and provide great flexibility in representing one or more networks of machines. But when attackers recognize a honeypot, it becomes useless. In this paper, we address issues related to detecting and "camouflaging" virtual honeypots, in particular Honeyd, which can emulate any size of network on physical machines. We find that an attacker may remotely fingerprint Honeyd by measuring the latency of the network links emulated by Honeyd. We analyze the threat from this fingerprint attack based on the Neyman-Pearson decision theory and find that this class of attack can achieve a high detection rate and low false alarm rate. In order to counter this fingerprint attack, we make virtual honeypots behave like their surrounding networks and blend in with their surroundings. We design a camouflaged Honeyd by revising a small part of the Honeyd toolkit code and by appropriately patching the operating system. Our experiments demonstrate the effectiveness of our approach to camouflaging Honeyd.
\end{abstract}

\section{INTRODUCTION}

Honeypots have recently been proposed as a means to complement the traditional defenses such as firewalls and Intrusion Detection Systems (IDS) for securing the computer networks that connect with the Internet. A honeypot is just a trap in cyberspace and no legal users are supposed to use it. Any activities taking place on it are inherently suspicious and likely a probe, attack or compromise. In this way, honeypots have few false alarms in detecting intrusions, while other IDS solutions often suffer from high false alarm rates since they are trying to isolate intrusions from within a high volume of routine network traffic.

Honeypots take different forms to target different situations. According to the level of interaction an attacker is allowed to have with a honeypot, there are low-interaction and highinteraction honeypots, respectively. Low-interaction honeypots restrict the interaction between an attacker and the honeypot. This effectively limits the scope of the attacker's actions and minimizes the risk of compromise. On the other hand, highinteraction honeypots expose the whole system, including the operating system, to an attacker. On high interaction systems, attackers can gain full control of the system.

Honeypots can also be categorized with respect to their implementations. A physical honeypot is a real machine with its own operating system and IP address, while a virtual honeypot is a machine which emulates system behavior and IP addresses. Virtual honeypots have a number of benefits: a single physical system can emulate more than one virtual honeypot; they can emulate more than one operating system; and they can emulate different IP addresses. Virtual honeypots require far less computational and network resources than physical honeypots, and they provide far greater flexibility in emulating various operating systems. In this research, we focus on studying virtual honeypots.

Honeyd [1] is a low-interaction virtual honeypot. It emulates computer systems at the network level. Honeyd emulates the TCP/IP stack and network services of an operating system in order to convince the attackers that they are exploring a "real" system. Honeyd can emulate network services such as HTTP and Telnet. It responds to a network request with response packets modified to match the network behavior of the operating system it has been configured to emulate. Besides emulating multiple services on multiple machines, Honeyd also emulates network topology and link characteristics such as link latency and packet loss.

To perform its function, a virtual honeypot such as Honeyd has to avoid being discovered. A virtual honeypot that cannot hide itself is analogous to a trap without any camouflage. In this paper, we will show that virtual honeypots such as honeyd may demonstrate distinct temporal signatures that make them easily detectable. By examining link latency within a network emulated by Honeyd, an attacker can determine whether the corresponding links are virtual or real. This stems from the fact that virtual honeypots are not typically designed to emulate the temporal behavior of nodes at high fidelity. Honeyd is not designed to support arbitrary link latency resolution. Ordinary operating systems, for which Honeyd is designed, typically do not support the necessary timing resolution either. As a result, the virtual honeypot displays a significantly different temporal signature from the physical systems and networks they are emulating, and the virtual link characteristics differ statistically from those of surrounding physical links. Using a campus network as an example, we analyze the threat from this fingerprint attack using Neyman-Pearson decision theory and find that this class of fingerprint attack can achieve a high detection rate with a low false alarm rate.

To counter this class of fingerprint attack against virtual honeypots such as Honeyd, we design a camouflaged Honeyd, which supports a link latency on the order of one microsecond instead of the original one millisecond default. Correspondingly, we generate a new patch to Linux 2.6.10 in order to support the new Honeyd implementation. Using the Texas A\&M University campus network as an example again, we demonstrate the effectiveness of our camouflage approach to hide Honeyd within a metropolitan network (MAN, a network comprised of multiple LANs). We develop the actual toolkits 
for camouflaging Honeyd and rewrite a small part of the original Honeyd toolkit code. These toolkits and instructions can be found in [2].

The rest of this paper is organized as follows. Section II presents the related work. We discuss the network model and threat model in Section III. In Section IV, we investigate how an attacker may discover Honeyd by measuring the link latency and evaluate its threat against Honeyd. In Section V, we discuss the necessary approaches to camouflage Honeyd from the attacker. We conclude this paper in Section VI.

\section{RELATED WORK}

In this section, we will survey various implementations of honeypots and then follow that by exploring work related to attacks and defenses for honeypots. Honeypots are classified into physical and virtual honeypots according to their implementation. In addition, they can be classified as low-interaction or high-interaction.

User-Mode Linux (UML) [3] is designed as a safe, secure way of running Linux versions and Linux processes on one machine. VMware Workstation [4] is powerful desktop virtualization software for software developers/testers and IT professionals who want to streamline software development, testing and deployment in their enterprise. Both UML and VMware can be used as virtual high interaction honeypots since they run full-fledged services on one machine.

Honeyd [1] is categorized as a low-interaction virtual honeypot and is implemented to emulate computer systems at the network level. In particular, Honeyd emulates the TCP/IP stack of a target operating system in order to convince attackers that they are exploring a "real" system. A Honeynet [5] is a highinteraction physical honeypot, a research effort originated by the Honeynet Project organization with the goal "to learn the tools, tactics, and motives of the blackhat community and share these lessons learned." A honeynet may contain one or more honeypots and is highly monitored because of the likelihood of being compromised.

Dornseif, Holz and Klein [6] presented an approach for attacking a honeynet locally when attackers break into a honeynet host. Two articles have been published on local honeypot identification in two issues of Phrack [7].

Kohno, Broido and claffy developed a method to fingerprint physical devices based on the skews of the devices' physical clocks [8]. That approach can be used to determine whether different addresses correspond to virtual hosts on the same physical machine. However, that approach would not work if the hosts being fingerprinted do not provide timestamps (e.g., with TCP timestamp option disabled). In this paper, we provide a complementary approach that does not rely on timestamp information from the target hosts.

In this paper, we will discuss techniques to measure link latency. Accurately measuring link latency is still a challenging research topic. A few network measurement tools (pathchar [9], clink [10], [11], pchar [12] and pipechar [13]) use a similar philosophy for the link latency measurement. Those tools utilize the field of TTL (time to live) in ICMP or UDP packets. We provide a complete set of approaches to measure link latency in our context, including the use of TCP packets.

\section{MODELS}

In this section, we introduce the network model and threat model used in this paper.

\section{A. Network Model}

Honeypots are decoys deployed in a network in order to trap attackers (and attack) and to learn their tools, tactics and motives. With this information, we can better understand and protect against threats. Figure 1(a) illustrates the network model for a virtual honeypot. The virtual honeypot can emulate either a single host or a network, whose setting and characteristics are configurable. We denote the emulated network as a virtual network. The virtual honeypot connects to the open network, such as the Internet, through a physical network interface. This physical interface can connect to a security gateway, which logs, monitors and limits activities on the virtual honeypot. In this way, the virtual honeypot is open to hacking, worms, spams and any other attack. This model is a general framework and can represent both high-interaction virtual honeypots such as User-Mode Linux (UML) and lowinteraction virtual honeypots such as Honeyd for the objective of intrusion detection.

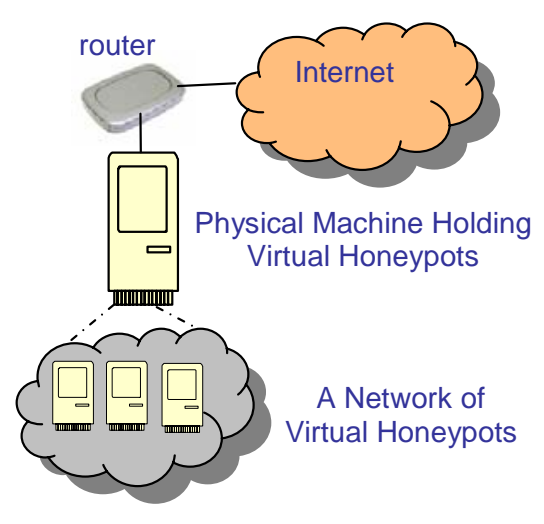

(a) Network Model

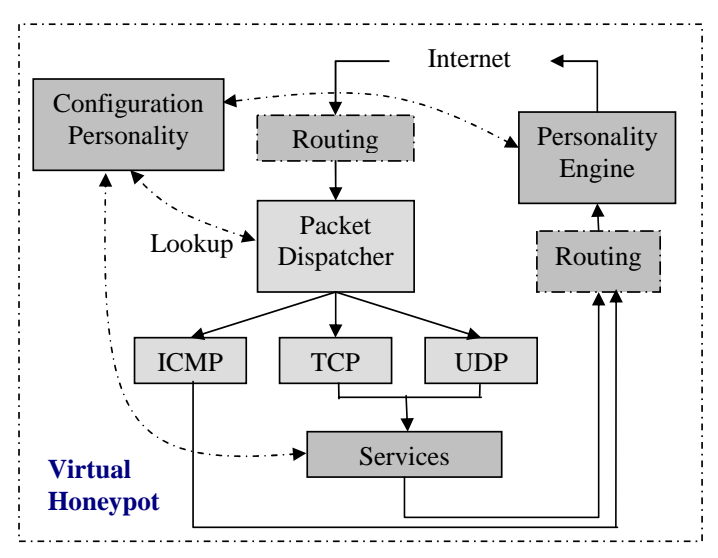

(b) Honeyd Infrastructure adapted from [1]

Fig. 1. Virtual Honeypot Models 
Virtual honeypots are the focus of this paper. Figure 1(b) illustrates the infrastructure of Honeyd. A virtual network emulated by Honeyd consists of a variety of components such as routers and end hosts. The emulated hosts may behave like a machine installed with Windows, Unix, Linux or other operating systems. The characteristics of these network units are delimited by specific scripts. Packets to the virtual network are processed in the following way: when the packet dispatcher receives a packet, it traverses the correct entry routing tree from the root node to the destination node. The packet latency on all edges of the path is accumulated to determine how long its delivery should be delayed. The packet delivery uses the event scheduling mechanism provided by libevent [14]. The libevent APIs are a wrapper of the underlying operating signal APIs and provide an alternative mechanism to support callbacks due to signals or regular timeouts.

\section{B. Threat Model}

Before we proceed, we need to clarify the capabilities of an attacker who is interested in determining whether a network is a virtual one created by honyed or a real physical one. (1) The attacker is an active one. They can inject probing traffic into the Internet remotely as normal users do. They can adjust the frequency and intensity of the probing traffic in order to prevent it from being discovered. (2) The attacker can utilize tools to discover the network topology [15]. Then, the attacker can choose one suspect link to test whether it is real or virtual. We will discuss how an attacker may use a tool to discover a metropolitan network topology. (3) The attacker knows the emulation strategies and algorithms of virtual honeypots. This assumption is commonly used in security research.

\section{RECOGNIZING Honeyd}

In this section, we first give an overview on how an attacker may fingerprint a virtual network emulated by Honeyd. We then define the problem formally and introduce issues related to the fingerprint attack. Because of the page limit, we will only show our major results as follows for recognizing and camouflaging honeyd. Please refer to the extended technical report [2] for details.

\section{A. Overview and Problem Definition}

Since large portions of the operational nodes (for example, users, server programs and network devices) are missing from virtual honeypots, emulating their operations requires triggering by timer events or signals. Packets to the virtual network are dispatched by timers. The accuracy of event scheduling is determined by the timing accuracy of the underlying operating system. In modern operating systems, timer interrupts are generated by the system's timing hardware at regular intervals. At boot time, the operating system kernel sets this interval according to the kernel parameter $H Z$, which is architecturedependent. Without loss of generality, in this paper, we focus on studying Honeyd deployed on Linux on x 86 PC. The results in this paper and our analysis approach are readily applied to other platforms.

HZ of the x86 PC defaults to 1000 in Linux kernel 2.6 and 100 in the previous versions of Linux kernel up to 2.4 [16].
These interrupt rates mean that the link latency emulated by systems like Honeyd can only achieve an accuracy of $10 \mathrm{~ms}$ and $1 \mathrm{~ms}$ for Linux 2.4 and 2.6 kernels respectively. Therefore, the link latency within a virtual network will always be around a multiple of $10 \mathrm{~ms}$ or $1 \mathrm{~ms}$. There are two security concerns raised by this observation: (1) a link latency with multiples of $10 \mathrm{~ms}$ or $1 \mathrm{~ms}$ can be a fingerprint of Honeyd; (2) with today's network hardware and bandwidth capacity, the link latency within a wired network can barely reach $1 \mathrm{~ms}$, let alone $10 \mathrm{~ms}$. This discrete latency gives rise to a timing signature of a virtual network emulated by Honeyd. Thus, if an attacker can recognize that a link exhibits such a signature, they can conclude that this is an emulated link and the corresponding network is virtual rather than physical.

Therefore, the problem of recognizing Honeyd can be defined as follows: Given a link connecting two routers within a network, how can an attacker determine by measuring the link latency whether the link is a real physical link or one emulated by Honeyd?

\section{B. Issues Related to Recognizing Virtual Honeypots}

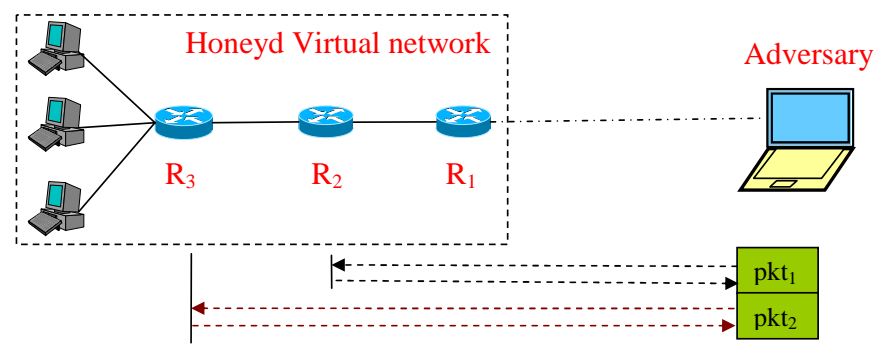

$2 \times($ Link Latency between R2 and R3 $)=\mathrm{RTT}_{\mathrm{pkt} 2}-\mathrm{RTT}_{\mathrm{pkt} 1}$

Fig. 2. Principle for recognizing Honeyd

From the discussion above, we know the success of recognizing Honeyd lies in accurately measuring link latency of a remote network. Figure 2 demonstrates the principle method of deriving link latency ${ }^{1}$. In Figure 2, RTT refers to Round Trip Time. To find the link latency of a network link connecting routers $R_{2}$ and $R_{3}$, an attacker can send a pair of packets $<p k t_{1}, p k t_{2}>$ of minimal size. $p k t_{1}$ is addressed to router $R_{2}$ while $p k t_{2}$ is addressed to router $R_{3}$. Intuitively,

$$
\begin{aligned}
\text { two-way link latency } & =2 \times L L_{R_{2}, R_{3}} \\
& =R T T_{p k t 2}-R T T_{p k t 1}
\end{aligned}
$$

where $R T T_{p k t n}$ is the RTT of packet $n$ and $L L_{R_{2}, R_{3}}$ is the one-way link latency between $R_{2}$ and $R_{3}$. In the following discussion, when we mention link latency, we refer to the twoway link latency for brevity.

Network noise may influence the accuracy of link latency measurement [10]. When a packet traverses the Internet, the intermediate routers and transmission media may disturb the timing of the packet. This forms the network noise, which disturbs the measurement of RTT. In our experiments, we use the same statistical analysis as the one used by clink [10],

\footnotetext{
${ }^{1}$ We use a virtual network topology similar to the one defined in config.sample, shipped with Honeyd 1.0 [17].
} 
[11] for deriving link latency ${ }^{2}$. Interested readers may refer to [10] for the details. Accurately measuring link latency is still a challenging research topic.

Therefore, from the discussion above, we can see that from the viewpoints of both attackers and defenders, there are four critical issues:

1) What kind of packets can the attacker utilize to derive the round trip times and the link latency?

2) How can an attacker recognize a virtual network using the measured link latency?

3) How effective is this form of fingerprint attack?

4) How can we hide Honeyd from this form of fingerprint attack?

We will answer these questions in the following sections.

\section{Measurement of Round Trip Times}

In this section, we answer the first question in Section IVB: "What kind of packets can the attacker utilize to derive the round trip times and the link latency?" The key to successfully recognizing a virtual network is to accurately derive the link latency. For the approach illustrated in Figure 2, an attacker has to measure the RTT of a packet sent from the attacker's probing machine to the victim router. This requires that the router generates a reply packet to a probing packet. The RTT can then be calculated as the difference between the sending time of the probing packet and the receiving time of the reply packet. There are several ways for an attacker to force a reply packet from nodes (routers or end hosts), depending on what services are provided on the node.

1) Ping Based Approach: This might be the most straightforward approach. The Ping program sends an ICMP echo request message to a host and expects an ICMP echo reply to be returned in order to check if a network unit is alive [18].

2) TCP Based Approach: There are two main classes of TCP based approaches to derive the RTT. First, if the victim host supports some well-known TCP services, the attacker can exploit these services to get the RTT. For example, if the victim supports Telnet, an attacker can exploit the procedure of establishing TCP connections (the three way hand shake). When an attacker sends a SYN packet to the victim router, the TCP service on a router or host will return an ACK/SYN packet in response to the attacker's SYN packet.

Even without any well-known TCP service on the victim router, the attacker may still be able to exploit the TCP protocol to derive the RTT. A reset (RST) is sent by TCP whenever an arriving TCP segment doesn't appear correct for the corresponding connection. Thus, the attacker can send a TCP packet with a bogus TCP source or destination port number to the victim router. Then the router will return a TCP RST packet to the attacker. Thus, the attacker obtains a reply packet and can calculate the RTT. We use this approach in our experiments. To correlate sent and received TCP packets, the attacker can utilize the TCP sequence number and acknowledgement number.

\footnotetext{
${ }^{2}$ clink utilizes UDP traffic as the probing traffic. We can also apply its measurement principle to ICMP and TCP traffic.
}

3) UDP Based Approach: Similarly, RTT information can be gathered with UDP as well. If the victim network unit supports some well-known UDP services, the attacker can exploit these services to get the RTT. If no such services are supported on the router, the attacker may still be able to exploit the UDP protocol to get RTT information. Similarly to the case of TCP, the victim router may respond to erroneous UDP packets. For example, when UDP receives a datagram and the destination port does not correspond to a port that some process is using, UDP responds with an ICMP "port unreachable" packet [18]. The attacker is also able to correlate a sent UDP packet to a received ICMP packet by using the source or destination port because the ICMP packet contains the UDP header. We use this approach in our experiments.

Finally, it must be pointed out that any of the methods mentioned above (and others) can be easily foiled by not having the virtual honeypot respond to the request or to the erroneous packet. This, however, would render the honeypot "silent", in which case it can only be used as a port knocking detector. In order for honeypots (physical or virtual) to be useful for gathering information about attacks, they must at least engage the attacker in some packet exchange, at which point some RTT information can be gathered.

\section{Recognizing Virtual Honeypots}

Once the RTT information has been gathered, and the link latency determined, a decision must be made on whether the link is part of a virtual honeypot. This section answers the second question in Section IV-B: "How can an attacker build a profile and recognize Honeyd based on the profile?"

1) Framework of Detecting Virtual Honeypots: The above problem is naturally a decision problem. Generally speaking, the goal of the pattern recognition process is to use classifiers to classify an unknown pattern as belonging to one of several existing patterns, classes, with the help of some distinguishing feature. In this paper, we use link latency as the feature and leave other features to exploit in our future work..

In the problem of detecting virtual honeypots, there are only two classes of events:

$$
\begin{aligned}
& \omega_{0}: \text { A suspect link is a real link } \\
& \omega_{1}: \text { A suspect link is a virtual link }
\end{aligned}
$$

Therefore, following the common practice, a pattern recognition system for detecting virtual honeypots consists of two phases: (a) off-line training and (b) on-line recognition.

Below is the procedure for the off-line training phase.

Collect training data from real networks and virtual networks: An attacker applies the probing traffic to known real networks and virtual networks. They collect the RTT data samples for both real links and virtual links.

Preprocess training data: The attacker derives two classes of link latency data sample from the RTT data by using approaches described in Section IV-C: real link latency sample and virtual link latency sample. From these two classes of link latency sample, the attacker is able to derive two distributions: real link latency distribution, denoted as $f_{0}(x)$, and virtual link latency distribution, denoted as $f_{1}(x)$. In this paper, we assume 
that the attacker uses kernel based density estimation approach [19], [20].

Select decision rule: The attacker selects an appropriate classification rule based on the training data and the two trained link latency distributions. In this paper, we assume that the attacker uses a classifier based on the Neyman Pearson theory, which will be introduced in the following section.

The procedure for the on-line recognition phase is similar to the off-line training phase. An attacker collects a link latency sample from a suspect network. Then the attacker uses the trained classifier to decide whether the link is real (class $\omega_{0}$ ) or virtual (class $\left.\omega_{1}\right)$.

2) Classifier Based on Neyman-Pearson Decision Theory: In this paper, we assume that an attacker uses a classifier based on the Neyman-Pearson (NP) decision theory [21] for recognizing virtual honeypots. The advantage of this theory is that Neyman-Pearson classification does not assume knowledge of or about a priori class probabilities such as those used in Bayesian classification theory.

We define detection rate $P_{D}$ as the probability that a link emulated by Honeyd is detected as a virtual link. False-alarm rate, $P_{F}$, is the probability that a real link is misclassified as a virtual link. We have the following Neyman-Pearson criterion:

Given a maximum false alarm rate $\alpha$, maximize detection rate $P_{D}$ such that $P_{F} \leq \alpha$

Neyman-Pearson criterion tells us that we should construct our decision rule to have the maximum detection rate while not allowing the false alarm rate to exceed a certain value $\alpha$. In other words, the optimal detector according to the Neyman-Pearson criterion is the solution to this constrained optimization problem in (4).

This optimization problem has an explicit solution. It is given by the Neyman-Pearson lemma. We limit our discussion to continuous random variables. Recall $f_{0}(x)$ as the link latency PDF of a real link and $f_{1}(x)$ as the link latency PDF of a link emulated by Honeyd.

Neyman-Pearson Lemma: Define

$$
\wedge(x)=\frac{f_{1}(x)}{f_{0}(x)}
$$

For a given maximum false alarm rate $\alpha$, we can calculate a decision boundary $\gamma^{\prime}$ by (6):

$$
P_{F}=\int_{\wedge(x)>\gamma^{\prime}} f_{0}(x) d x=\alpha
$$

Then we have the following classifier

$$
\wedge(x)=\frac{f_{1}(x)}{f_{0}(x)}\left\{\begin{array}{cl}
<\gamma^{\prime}, & \text { the suspect link is real } \\
>\gamma^{\prime}, & \text { the suspect link is virtual }
\end{array}\right.
$$

Detection rate can then be calculated as follows:

$$
P_{D}=\int_{\wedge(x)>\gamma^{\prime}} f_{1}(x) d x
$$

Please note that here we assume that the mean of $f_{1}(x)$ is greater than the mean of $f_{0}(x)$. This assumption will be validated from our experimental results.

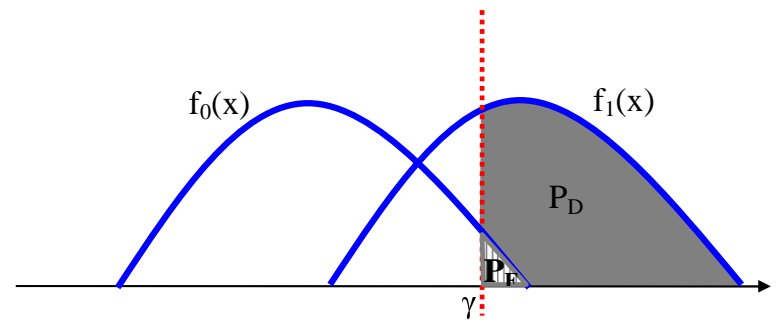

Fig. 3. Calculation of $P_{D}$ and $P_{F}$

When we use a classifier based on the Neyman-Pearson theory, because we know $f_{0}(x)$ and $f_{1}(x)$, the classifier in (7) is often translated, either theoretically or numerically, to a simpler classifier as shown in (9):

$$
x \begin{cases}<\gamma, & \text { the suspect link is real } \\ >\gamma, & \text { the suspect link is virtual }\end{cases}
$$

We will use (9) as the classifier in this paper. Figure 3 illustrates how to calculate detection rate $P_{D}$ and false alarm rate $P_{F}$ in this paper, where $\gamma$ is the derived decision boundary.

We implemented the above detection rate and false alarm rate calculation in Matlab.

\section{E. Evaluation}

In this subsection, we evaluate the threat from an attacker who applies Neyman-Pearson decision theory to distinguish a Honeyd emulated virtual link from a real link.

1) Evaluation Metrics: In this paper, we use detection rate $P_{D}$ and false alarm rate $P_{F}$ as our evaluation metrics for detecting honeyd. From Figure 3, we can see that detection rate $P_{D}$ and false alarm rate $P_{F}$ have an interesting relationship. Both $P_{D}$ and $P_{F}$ decrease to zero as $\gamma$ increases, while both $P_{D}$ and $P_{F}$ increase to one as $\gamma$ decreases. To display the relationship between $P_{D}$ and $P_{F}$, we use a Receiver Operating Characteristic $(R O C)$ curve, which is a plot of $P_{D}$ versus $P_{F}$. In reality, when an attacker tries to find a virtual honeypot by using the Neyman-Pearson theory, they want a high detection rate and a low false alarm rate. If we want to hide virtual honeypots, the ideal scenario is an attacker will get a false alarm rate as high as the detection rate.

2) Experiment Setup: Figure 4 illustrates our experimental setup. We deploy a virtual Honeyd network in our laboratory ${ }^{3}$. The objective of the attacker is to decide whether the link between $R_{2}$ and $R_{3}$ is a Honeyd emulated link (the same configuration as the one in Figure 2. We have similar results for other virtual network topologies). Between the Honeyd virtual network and the attacker's machines, we set up a machine with NISTnet [22] to emulate the influence from cross traffic or intermediate networks on the detection of Honeyd.

3) Deriving Link Latency Distributions: In order to detect a Honeyd emulated link, the attacker will use the pattern recognition based approach described above. During offline training, attackers will derive the latency PDFs for both real

\footnotetext{
${ }^{3}$ Because of the university administration issues, we cannot deploy Honeyd at a campus scale.
} 


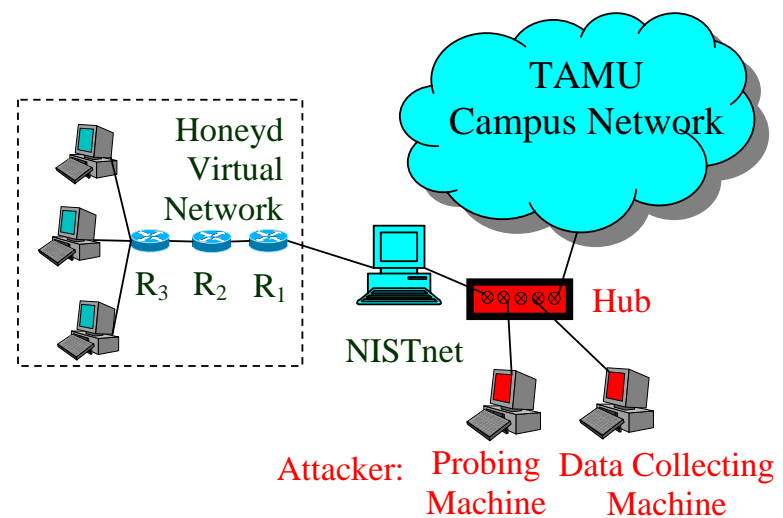

Fig. 4. Experiment Setup

and virtual links. To derive the link latency distributions for the real surrounding network links, an attacker can first derive the network topology of the target network and then use one of the approaches in Section IV to derive the link latency of all the links. Then, from the measured link latency samples, the attacker can derive the link latency distribution for the network. Recall the attacker uses the Gaussian Kernel based density estimation approach to derive the empirical density function in this paper, .

In our experiments, we use a network topology discovery tool called Argus [23]. This tool uses an ICMP/ traceroutebased topology discovery methodology. It is flexible and can be used for most networks. We applied Argus against Texas A\&M University's campus network and found nearly 20,000 running network units. Figure 5 shows the real link latency distribution. Negative values in the distribution are caused by the kernel based density estimation approach and can be ignored.

To derive link latency distributions of Honeyd emulated links, the attacker can use the setup in Figure 4 and derive link latency distributions for Linux 2.4 and 2.6 kernels. Figure 6 demonstrates the probability density function (PDF) of link latency when an attacker uses the TCP probing approach.

4) Failure of Current Honeyd Implementation: We have a few observations from Figure 5 and Figure 6: (1) We can see that the link latency emulated by Honeyd is roughly a multiple of $10 \mathrm{~ms}$ on Linux 2.4.18 and $1 \mathrm{~ms}$ on Linux 2.6.9, as we analyzed in Section IV-A. Clearly, the mean of each virtual link latency density function is larger than the mean of the real link latency distribution. This validates the assumption made in Section IV-D.2. It is a quirk of OS and honeyd scheduling policies that Linux 2.6.9 yields a factor of one times the default interrupt rate while Linux 2.4.18 yields a factor of two times the default interrupt rate. (2) Moreover, the PDF curve for virtual link latencies is confined to a very narrow range. This means that a packet pair based probing approach in this case generates very accurate measurement of link latency. (3) The real campus network link latency distribution in Figure 5 and Honeyd-emulated network link latency in Figure 6 are widely different along the axis of link latency.

The above observations can well explain the ROC curves in Figure 7 in the case of detecting a virtual link on Linux
2.6.9. From Figure 7, we have the following observations: An attacker is able to achieve a high detection rate while keeping the false alarm rate low for any of the TCP, UDP or Ping based approaches. For example, when the false alarm rate is around $2 \%$, an attacker may achieve a detection rate of over $98 \%$ in all three cases. The reason is the Honeyd-emulated network link latency is much larger than the real network link latency. Moreover, the emulated link latency is narrowly confined to a region around $1 \mathrm{~ms}$ or $20 \mathrm{~ms}$.

TCP, UDP and Ping probes produce similar ROC curves. We expect this since our experiments are run within a campus network. If an attacker deploys the attack from a remote site, TCP and UDP probes will be able to achieve better performance because the Ping packets' low scheduling priority on the Internet. In the remaining part of this paper, we will not differentiate results from TCP, UDP and ICMP probes.

\section{Camouflaging Honeyd}

The key reason for the failure of the current Honeyd implementation (and likely other virtual honeypot implementations as well) is the low fidelity of emulation (in the time domain) of the system components by the virtual honeypot. These components may represent users, applications and servers in the case of physical honeypots, in addition to various hardware resources in the case of virtual honeypots. The processing delay caused by these components is emulated by timers in the virtual honeypot. When these timers are either carelessly defined in the virtual honeypot implementation or are provided at insufficient accuracy by the underlying OS, a timing signature emerges. In this case, an attacker may construct a profile of a virtual honeypot and launch a timing attack. In the case of Honeyd, for example, emulated virtual links are statistically very different from the surrounding physical network links.

\section{A. Overview of the Principle}

To camouflage Honeyd and defeat the type of timing attacks described above, we have to modify Honeyd and the underlying OS support to allow for a higher-fidelity emulation of events. This requires (a) configurable definition of accurate timing behavior, and (b) support for accurate triggering of events by the underlying OS. In our case, this means (a) accurately configurable link latencies, and (b) high-resolution timers within the OS. In this way, we can assign a reasonable link latency for Honeyd based on its surrounding network link characteristics, and an attacker will not be able to build a useful profile for Honeyd.

We camouflage Honeyd in the following ways: (1) We change the Honeyd code to make it support a timing resolution of microseconds $\mu s$. This involves modifying both Honeyd and the event management library (libevent). (2) There are a number of ways to improve the OS support for high-fidelity timers. We can use the real-time event scheduling in commercial real-time operating systems [24] or one of various open source projects for high resolution timers [25]. The advantage of this approach is that we may achieve high-resolution timers at low overhead since these approaches typically make use of hardware support for accurate timers. The disadvantage is 


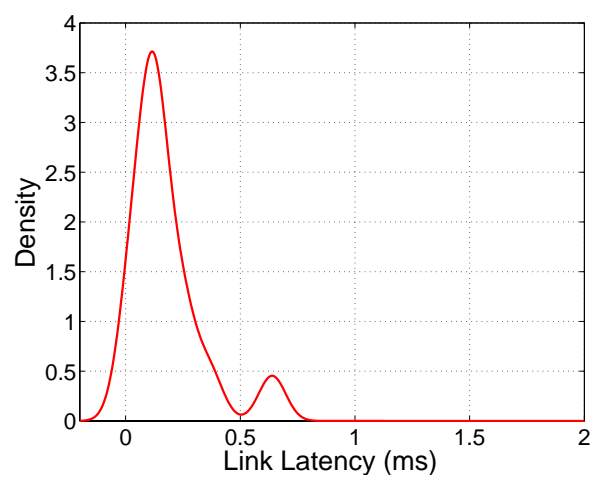

Fig. 5. Campus Network Link Latency Distribution

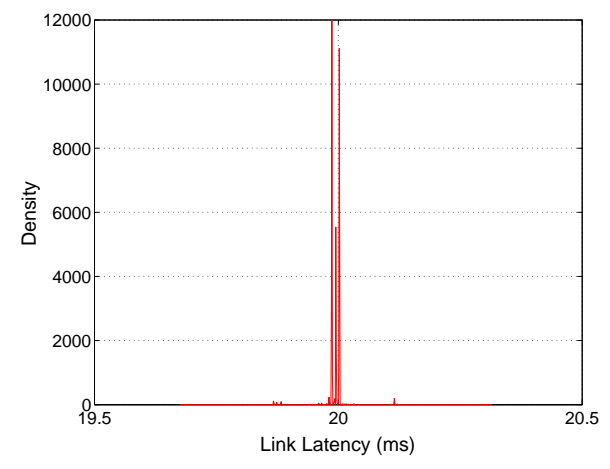

(a) Link 2.4.18

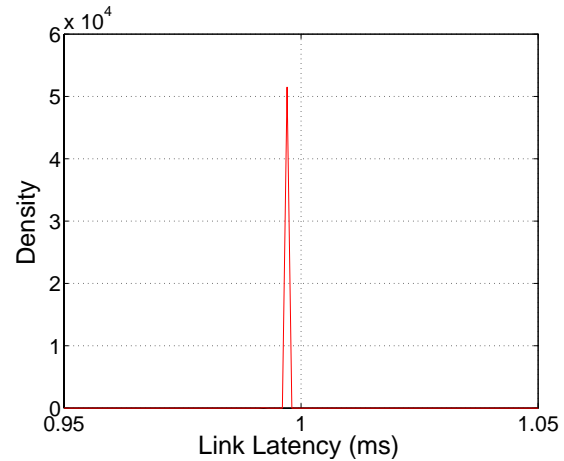

(b) Linux 2.6.9

Fig. 6. PDFs of Virtual Link Latency (Note the finer $x$-axis scale compared with Figure 5)

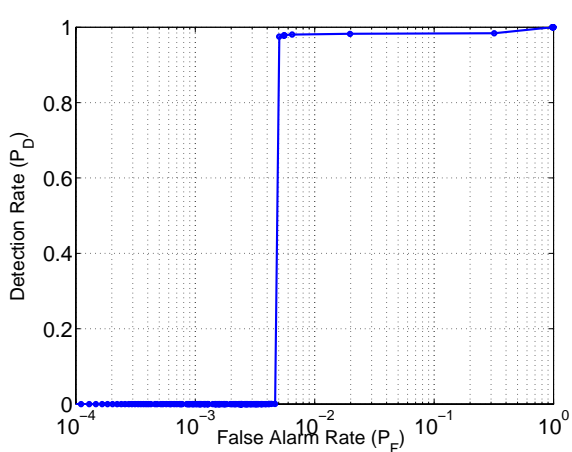

(a) TCP

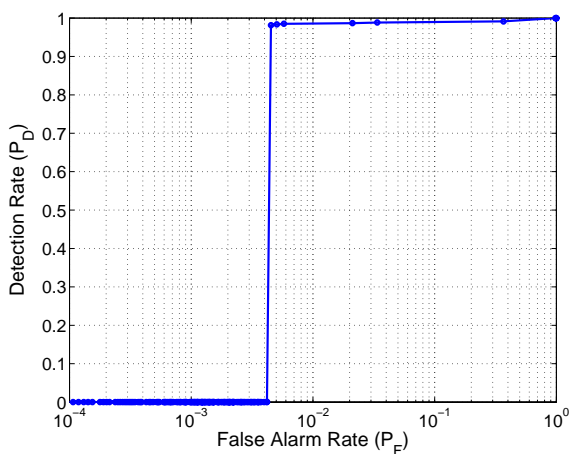

(b) UDP

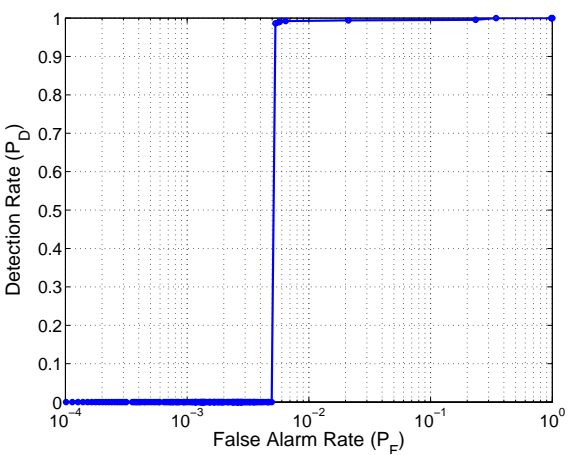

(c) Ping

Fig. 7. ROC for Fingerprint Attack

that we would need to rewrite the event management library (libevent). In this paper, we improve the operating system's timing accuracy by increasing the kernel parameter $H Z$ (Refer to Section IV-A). We ported the HZ patch [26] to kernel 2.6.10. In this way, we don't need to change the libevent library at all, however we may sacrifice some CPU efficiency. Since the physical machine running Honeyd should not be running any other applications, this is not crucial.

Thus, if the PDF of the Honeyd emulated link latency can approach the real network link latency PDF in Figure 5 , an attacker cannot determine whether a link is emulated by Honeyd or not. In the following, we demonstrate the effectiveness of camouflaging virtual honeypots.

\section{B. Effectiveness of Camouflaging}

Recall that in order to camouflage Honeyd from the fingerprint attack as discussed in this paper, we need to make Honeyd capable of emulating real network link latency. For example, in the context of the Texas A\&M University campus network, if Honeyd emulated link latency could have a distribution like the one in Figure 5, the attacker will not be able to use the fingerprint attack to tell the difference between emulated links and real links. Therefore, in order to camouflage Honeyd, we determine a link latency distribution for the virtual network that is as close as we can emulate to the real network's link latency distribution. Then we randomly generate values from our approximate distribution to use when we configure Honeyd emulated link latencies.

From the above discussion, we know that if the PDF of the Honeyd emulated link latency can approach the real network link latency PDF in Figure 5, an attacker cannot determine whether a link is emulated by Honeyd or not. Figure 8 shows the emulated network link distribution we generate to match the real campus network latency distribution. We can see the similarity of the two distributions in Figure 8. In our experiments, we set $\mathrm{HZ}$ as 20,000 and adjust link latency in the Honeyd configuration. With few adjustments, we can achieve the effect shown in Figure 8. Actually, since we can adjust parameters $\mathrm{HZ}$ in the Linux kernel and link latency in the Honeyd configuration, we may produce a broad range of distribution patterns resembling real network link latency distributions.

Figure 9 shows the curve of detection rate and false alarm rate versus decision boundary. From Figure 9, we can derive Figure 10, which shows the ROC curve for the camouflaged Honeyd. The ROC curve almost follows a 45 degree diagonal, which represents the worst-case for detection, but the best case for camouflaging. That is, when the detection rate is big, the false alarm rate is big. When the detection rate is small, the false alarm rate is small too. For example, our experiments show that when the detection rate is $97 \%$, the false alarm rate is as high as $90 \%$. When the detection rate is $15 \%$, the false alarm rate is around $27 \%$. This shows that when we apply the above camouflaging approach to Honeyd, an attacker can no 


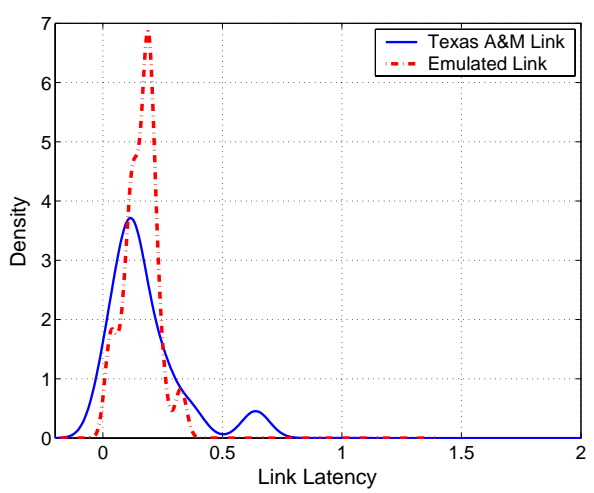

Fig. 8. Emulated Network Link Latency Distribu- Fig. 9. Detection Rate and False Alarm Rate vs tion v.s. Real Campus Network Latency Distribution Decision Boundary
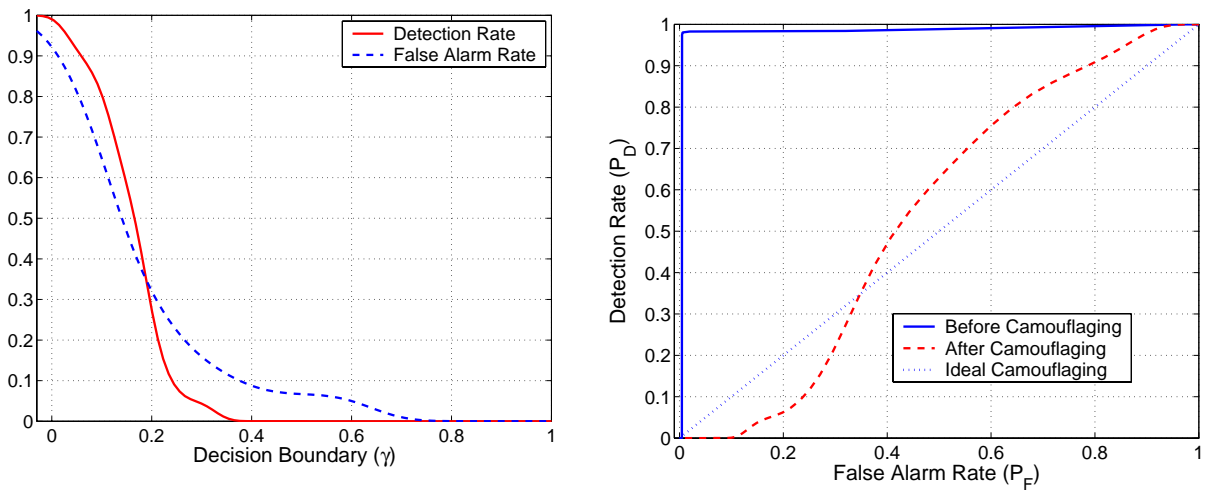

Fig. 10. ROC for Camouflaged Honeyd longer use the fingerprint attack discussed in this paper. This is an intuitive result considering the two overlapping distributions in Figure 8.

\section{CONCLUSIONS}

In this paper, we address issues related to detecting and "camouflaging" virtual honeypots. In general, virtual honeypots emulate characteristics of the target operating systems or networks. It is well known that such emulation is often a challenging task. An inappropriate emulation may expose the existence of the virtual honeypot. An attacker may find that the characteristics of a victim network cannot match believable parameters or those of its surrounding networks. We emphasize that great care has to be taken to hide virtual honeypots within their surroundings.

In particular, we find that Honeyd can neither configure nor emulate network link latencies at a resolution finer than $1 \mathrm{~ms}$. Our measured results demonstrated an emulation accuracy of $1 \mathrm{~ms}$ or $10 \mathrm{~ms}$ on a Linux platform, which is too large to accurately emulate a metropolitan network where Honeyd might be deployed. An attacker can build a profile of Honeyd link latency and remotely fingerprint Honeyd by measuring the link latency. Our experiments show that the attacker may derive a high detection rate for Honeyd with a low false alarm rate. To camouflage virtual honeypots, the key is to make them behave like the surrounding network. In our specific case, if the PDF of the Honeyd emulated link latency can approach the real network link latency PDF, an attacker cannot determine whether a link is emulated by Honeyd or not. To achieve a high fidelity emulation of link latency, we improve the OS timing accuracy by changing the Linux kernel parameter HZ.

For future work, we believe that other vulnerabilities exist in emulation based virtual honeypots and plan to do a thorough investigation of them and design compensating camouflaging approaches.

\section{REFERENCES}

[1] N. Provos, "A virtual honeypot framework," in Proceedings of USENIX Security Symposium, August 2004.

[2] X. Fu, W. Yu, D. Cheng, X. Tan, and S. Graham, "On recognizing virtual honeypots and countermeasures," http://www.homepages.dsu.edu/fux/, 2006.
[3] J. Dike, "The user-mode linux kernel home page," http //user-mode-linux.sourceforge.net/, 2005.

[4] vmware.com, "Vmware workstation 5," http://www.vmware.com/ products/desktop/ws_features.html, 2005.

[5] honeynet.org, "Honeynet project," http://www.honeynet.org/index.html, 2005.

[6] M. Dornseif, T. Holz, and C. N. Klein, "Nosebreak - attacking honeynets," in Proceedings of IEEE Workshop on Information Assurance and Security, June 2004

[7] phrack.org, "Phrack," http://www.phrack.org/, 2005.

[8] T. Kohno, A. Broido, and kc claffy, "Remote physical device fingerprinting," in Proceedings of the IEEE Symposium on Security and Privacy, May 2005.

[9] V. Jacobson, "Pathchar," http://www.caida.org/tools/utilities/others/ pathchar/, 1997

[10] A. B. Downey, "Using pathchar to estimate internet link characteristics," in Proceedings of ACM SIGCOMM, September 1999.

[11] _ _Clink: a tool for estimating internet link characteristics," http: //allendowney.com/research/clink/, 1999.

[12] B. A. Mah, "pchar: A tool for measuring internet path characteristics," http://www.kitchenlab.org/www/bmah/Software/pchar/, 2005.

[13] Computational Research Division, Lawrence Berkeley National Laboratory, "Network characterization service (NCS)," http://www-didc.lbl. gov/NCS/, 2005.

[14] N. Provos, "libevent - an event notification library," http://www.monkey. org/ provos/libevent/, 2004.

[15] B. Lowekamp, D. R. OHallaron, and T. R. Gross, "Topology discovery for large ethernet networks," in Proceedings of ACM SIGCOMM, August 2001.

[16] J. Corbet, A. Rubini, and G. Kroah-Hartman, Linux Device Drivers, Third Edition. O'Reilly, 2005.

[17] N. Provos, "Developments of the honeyd virtual honeypot," http://www. honeyd.org/, 2005.

[18] W. R. Stevens, TCP/IP Illustrated, Volume 1 The Protocols. AddisonWesley Professional, 1999.

[19] B. Silverman, Density Estimation for Statistics and Data Analysis, Monographs on Statistics and Applied Probability. London: Chapman \& Hall, 1986.

[20] C. Beardah, "Kernel density estimation toolbox," http://web.ccr.jussieu. fr/ccr/Documentation/Calcul/matlab5v11/docs/00053/05375.htm, 1999.

[21] R. O. Duda and P. E. Hart, Pattern Classification. John Wiley \& Sons, 2001.

[22] M. Carson and D. Santay, "Nist net - a linux-based network emulation tool," Computer Communication Review, vol. 33, no. 3, July 2003.

[23] S. Keshav, C. Estan, H. Chan, and W. Chang, "Project argus network topology discovery, monitoring, history, and visualization," http://www.cs.cornell.edu/boom/1999sp/projects/Network\%20Topology/ topology.html, 1999.

[24] TimeSys Corp. , "Timesys," http://www.timesys.com/, 2005.

[25] G. Anzinger, "This is the home page for the high resolution timer project," http://high-res-timers.sourceforge.net/, 2005.

[26] J.-M. Valin, "Increasing hz (patch for hz > 1000)," http://kerneltrap.org/ node/1766, 2003. 